\title{
Knockdown of ACTA2-AS1 promotes liver cancer cell proliferation, migration and invasion
}

\author{
RU-JIAN ZHOU* and HUI-ZENG LV* \\ Department of Forensic Surgery, The Fifth Affiliated Hospital of Guangzhou Medical University, \\ Guangzhou, Guangdong 510700, P.R. China
}

Received March 9, 2018; Accepted November 2, 2018

DOI: $10.3892 / \mathrm{mmr} .2019 .9856$

\begin{abstract}
Long noncoding RNAs (lncRNAs) are important regulators of various cellular and biological processes. The present study aimed to investigate the functions of a novel lncRNA, ACTA2-AS1:4, a transcript variant of smooth muscle $\alpha$-actin 2-antisense 1 (ACTA2-AS1), in regulating liver cancer progression. Expression of lncRNAs in liver cancer tissues and cell lines were analyzed by reverse transcription quantitative polymerase chain reaction (RT-qPCR). Knockdown of ACTA2-AS1:4 expression in LM3 liver cancer cells was achieved by transfection with small interfering RNAs (siRNAs) that specifically targeted ACTA2-AS1:4. The proliferation and cell cycle progression of ACTA2-AS1:4-silenced LM3 cells were determined using MTS assay and flow cytometry, respectively. A Transwell system assay was used to evaluate the migration and invasion capacities of LM3 cells transfected with ACTA2-AS1:4 siRNA. The expression levels of major genes associated with important cellular processes were finally determined by RT-qPCR and western blot analysis. ACTA2-AS1:4 expression in liver cancer tissues and multiple cell lines was markedly downregulated by specific siRNAs. This inhibition of ACTA2-AS1:4 expression significantly promoted the proliferation, cell cycle progression, migration and invasion of LM3 cells. A decrease in ACTA2-AS1:4 expression also suppressed E-cadherin expression, increased $\mathrm{N}$-cadherin expression, decreased caspase 3 expression and increased cyclin D1 and matrix metalloproteinase expression in liver cancer cells. Downregulation of ACTA2-AS1:4 affects a number of key mechanisms involved in liver cancer
\end{abstract}

Correspondence to: Dr Hui-Zeng Lv, Department of Forensic Surgery, The Fifth Affiliated Hospital of Guangzhou Medical University, 621 Gangwan Road, Huangpu, Guangzhou, Guangdong 510700, P.R. China

E-mail: 1vhuizengsci@sina.com

*Contributed equally

Key words: smooth muscle $\alpha$-actin 2-antisense 1, liver cancer, long non-coding RNAs, proliferation, migration, invasion progression. These data may be important for the future of liver cancer diagnosis and subsequent treatments.

\section{Introduction}

Liver cancer, also known as hepatic cancer, is a severe human malignant tumor; it is considered one of the major causes of cancer-associated mortality worldwide (1). Previous epidemiological evidence indicates increasing global rates of incidence and mortality of hepatic cancer, with particularly high incidence rates in East Asian countries including China $(2,3)$. The most common liver cancer subtype is hepatocellular carcinoma (HCC), which accounts for $>80 \%$ of cases of malignant liver tumors. The causative factors of HCC include chronic hepatitis $\mathrm{B}$ or $\mathrm{C}$ virus infection, hemochromatosis and excessive alcohol consumption $(2,3)$. The treatment options available include surgery, radiation therapy and targeted therapy; however, ideal therapeutic outcomes are primarily witnessed only in patients with early-stage cancer (3). Over the previous decades, progress in determining the molecular mechanisms of cancer progression have provided a foundation for the development of specific reagents that target cancer cells and also promising novel treatment strategies including immunotherapy (4) However, the majority of these strategies remain unsuitable for clinical application, partially due to an incomplete understanding of the complex pathological processes involved in liver cancer progression.

Long noncoding RNAs (lncRNAs), defined as transcripts $>200$ nucleotides but not used for translation of proteins in cells, have previously been revealed to be involved in various cellular processes and liver cancer tumorigenesis (5). Previously, genome-wide association studies of large cancer data sets revealed that major single-nucleotide polymorphisms were associated with noncoding genes; IncRNAs have been demonstrated to be a novel class of key noncoding factors in tumor development and progression (6). A large number of studies have suggested that lncRNAs perform their various biological and pathological roles by affecting gene transcription, translation and other molecular processes through interacting with DNA, RNA or protein components (7-10). This interaction has been indicated to be dependent on the origin of lncRNAs within the genome and also their structure $(11,12)$. For example, a number of IncRNAs are mediated by their ability to form various 3-dimensional structures and bind different 
protein components. For example, lncRNA-p21 is important for the localization of heterogeneous nuclear ribonucleoprotein complex $\mathrm{H}$ to specific promoters, resulting in global suppression of gene expression (13). At present, a number of lncRNAs have been demonstrated to be associated with the initiation and progression of liver cancer including highly upregulated in liver cancer (HULC) (14), HOX transcript antisense intergenic RNA (HOTAIR) (15), H19 (16), high expression in HCC (HEIH) (17), and metastasis-associated lung adenocarcinoma transcript 1 (MALAT1) (18). An additional study has indicated that IncRNAs may regulate liver cancer progression through epigenetic alterations, including DNA methylation and histone modifications (12). These IncRNAs have also been considered as biomarkers for early liver cancer detection and also as potential targets for developing combination therapies.

The smooth muscle $\alpha$-actin 2 (ACTA2) gene encodes smooth muscle-specific $\alpha$-actin and functions as a key contractile component of vascular smooth muscle. It has been associated with the development of various human diseases including aortic aneurysm, stroke, coronary artery disease, Moyamoya disease and multi-systemic smooth muscle dysfunction syndrome (19). ACTA2 has previously been indicated to be expressed as a member of Wnt-induced gene signature in human HCC and is associated with poor clinical outcomes (20). The lncRNA ACTA2 antisense RNA 1 (ACTA2-AS1) encoded by ACTA2, also known as ZXF1, was identified to be associated with cell invasion and metastasis in lung adenocarcinoma (21). ACTA2-AS1 has also been demonstrated to have significantly different expression patterns in human lung cancer tissues compared with adjacent normal lung tissues, and suppression of ACTA2-AS1 expression by small interfering RNAs (siRNAs) greatly inhibited lung cancer cell migration and invasion (21). The expression of ACTA2-AS1 in liver carcinomas and its role in liver cancer development and progression remain to be investigated.

The present study addressed the potential role of ACTA2-AS1:4, one of the transcript variantss of ACTA2-AS1, in liver cancer development by analyzing its expression in liver cancer tissues and also multiple liver cancer cell lines. Furthermore, ACTA2-AS1 was inhibited using specific siRNAs to investigate the role of ACTA2-AS1 in regulating liver cancer cell proliferation, invasion and migration. This provided important information for understanding the function of lncRNAs during hepatic tumorigenesis.

\section{Materials and methods}

HCC biopsies and cultured cell lines. Biopsies of cancerous tissues and paired healthy adjacent hepatic tissues were collected from 17 patients with HCC; these patients were hospitalized in the Department of Hepatobiliary Surgery, Second Affiliated Hospital of Sun Yat-sen University from January 2017 to December 2017. The study proceeded following the receipt of written informed consent from the patients, and tissue collection and subsequent experiments were approved by the Ethics Committee of the Sun Yat-sen University and the Fifth Affiliated Hospital of Guangzhou Medical University. Clinical samples were independently verified by 2 experienced clinical pathologists. Liver cancer cell lines, LM3, MHCC-97L, and MHCC-97L, and the normal liver cell line, L02, were obtained from the American Type Culture Collection (Manassas, VA, USA). Liver cancer HepG2 and Hep3B cell lines were purchased from the Type Culture Collection of the Cell Bank of Chinese Academy of Sciences (Shanghai, China). For the analysis of gene expression and cellular function, liver cancer cells were cultured in Dulbecco's modified Eagle's medium (Thermo Fisher Scientific, Inc., Waltham, MA, USA) containing $0.1 \%$ fetal bovine serum (FBS; Thermo Fisher Scientific, Inc.) and antibiotics at $37^{\circ} \mathrm{C}$ in a humidified incubator supplied with $5 \% \mathrm{CO}_{2}$.

Reverse transcription quantitative polymerase chain reaction $(R T-q P C R)$. For the determination of relative lncRNA levels, an RT-qPCR assay was performed using specific primers. HCC biopsies were stored in liquid nitrogen and fully homogenized using a mortar and pestle to extract RNA. To extract RNA from cultured liver cell lines and normal hepatic cells, cells were washed 3 times with PBS and centrifuged at $800 \mathrm{x}$ g for $5 \mathrm{~min}$ at $4^{\circ} \mathrm{C}$. The PureLink ${ }^{\mathrm{TM}}$ RNA Mini kit (cat. no. 12183018A; Thermo Fisher Scientific, Inc.) was used to extract RNA from liver cancer tissues and cultured cell lines, according to the manufacturer's protocol. RNA concentrations in each sample was determined using a spectrophotometer, and $\sim 1.5 \mu \mathrm{g}$ RNA from each sample was used to synthesize cDNA using the High-Capacity cDNA Reverse Transcription kit (cat. no. 4368813; Thermo Fisher Scientific, Inc.), according to the manufacturer's protocol. To quantitatively assess lncRNA expression, qPCR was performed using a SYBR Select Master Mix kit (cat. no. 4472908; Applied Biosystems; Thermo Fisher Scientific, Inc.), according to the manufacturer's protocol. To perform RT, the thermocycling conditions were as follows: $25^{\circ} \mathrm{C}$ for $10 \mathrm{~min}, 37^{\circ} \mathrm{C}$ for $120 \mathrm{~min}$ and $85^{\circ} \mathrm{C}$ for $5 \mathrm{~min}$. The PCR program were set as follows: $95^{\circ} \mathrm{C}$ for $30 \mathrm{sec}, 95^{\circ} \mathrm{C}$ for $10 \mathrm{sec}, 60^{\circ} \mathrm{C}$ for $30 \mathrm{sec}$, for 40 cycles. Then dissolution curve was generated by setting the program as $95^{\circ} \mathrm{C}$ for $15 \mathrm{sec}, 60^{\circ} \mathrm{C}$ for $60 \mathrm{sec}$ and $95^{\circ} \mathrm{C}$ for $15 \mathrm{sec}$. Data were analyzed using $2^{-\Delta \Delta \mathrm{Cq}}$ method (22). GAPDH served as the internal control for quantitation, and at least 3 independent repeats were performed for statistical analysis. Sequences of primers used in the present study were listed in Table I.

Transfection of ACTA2-AS1:4. The expression of ACTA2-AS1:4 was suppressed in LM3 cells, as previously described, with minor modifications (17). These are described as follows. The ACTA2-AS1:4 small interfering RNA (siRNAs; sense, 5'-GCCUGGUGGUAA AUAUGA ATT-3' and antisense, 5'-UUCAUAUUUACCACCAGGCTT-3') were synthesized by Shanghai GenePharma Co., Ltd. (Shanghai, China). LM3 cells, at $80 \%$ confluence, were transfected with the aforementioned siRNAs (200 nM) using Lipofectamine 2000 (Invitrogen; Thermo Fisher Scientific, Inc.), according to the manufacturer's protocol. Total RNA was extracted $48 \mathrm{~h}$ after transfection, and RT-qPCR was performed using this RNA. LM3 cells treated with Lipofectamine 2000 but not transfected with ACTA2-AS1:4 siRNA were used as the negative control (NC) for the expression inhibition assay.

Cell proliferation and viability assays. The viability of liver cancer cells was evaluated by assessing cell proliferation using the colorimetric MTS Cell Proliferation Assay kit 
Table I. Primers used in the present study.

\begin{tabular}{ll}
\hline Primer name & \multicolumn{1}{c}{ Sequence (5'-3') } \\
\hline lnc-MUC20-9-F & GCCCACCAAGTAAGGAACCC \\
lnc-MUC20-9-R & AAATCCAGGCCAGACCAATCT \\
ACTA2-AS1:4-F & GCCCATCAGGCAACTCGTAA \\
ACTA2-AS1:4-R & TCTTTGCCACCTGTGCAGAC \\
TRPM2-AS:1-F & CCCGAGGAAGGCTACTGATG \\
TRPM2-AS:1-R & GGCTGAGTGACGAGAAGCAA \\
H-GAPDH-F & GAGTCAACGGATTTGGTCGT \\
H-GAPDH-R & GACAAGCTTCCCGTTCTCAG \\
H-E-Cadherine-F & GAGAAACAGGATGGCTGAAGG \\
H-E-Cadherine-R & TGAGGATGGTGTAAGCGATGG \\
H-N-Cad-F & ATGAAAGACCCATCCACGC \\
H-N-Cad-R & CCTGCTCACCACCACTAC \\
H-MMP2-F & TGATGGCATCGCTCAGATCC \\
H-MMP2-R & GGCCTCGTATACCGCATCAA \\
H-Caspase3-F & CATGGAAGCGAATCAATGGACT \\
H-Caspase3-R & CTGTACCAGACCGAGATGTCA \\
H-CyclinD1-F & GCTGCGAAGTGGAAACCATC \\
H-CyclinD1-R & CCTCCTTCTGCACACATTTGAA
\end{tabular}

ACTA2, smooth muscle alpha-actin 2; Cad, cadherin; F, forward; $\mathrm{R}$, reverse; MMP2, matrix metalloproteinase 2; MUC20, mucin 20; TRPM2, transient receptor potential cation channel subfamily $\mathrm{M}$.

(cat. no. 197010; Abcam, Cambridge, MA, USA), according to the manufacturer's protocol. Briefly, LM3 cells and the control L02 cells were cultured in 96-well microtiter plates to $\sim 80 \%$ confluence and then incubated with $20 \mu \mathrm{g}$ MTS reagents for $2 \mathrm{~h}$ at $37^{\circ} \mathrm{C}$ in a humidified culture chamber supplied with $5 \% \mathrm{CO}_{2}$. The optical density values were measured at $490 \mathrm{~nm}$ using a plate reader and used for evaluation of cell proliferation and viability. For statistical analysis, at least three replicates were performed.

Cell cycle progression analysis. Cell cycle progression was evaluated by assessing the percentage of LM3 cells at G1, S, and G2/M stages following transfection. Briefly, LM3 cells were washed 3 times with PBS, fixed with $70 \%$ ethanol overnight at $20^{\circ} \mathrm{C}$, stained with $\sim 300 \mu \mathrm{l}$ propidium iodide (Hangzhou MultiSciences(Lianke)Biotech, Co., Ltd., Hangzhou, China) in the dark at $37^{\circ} \mathrm{C}$ for $30 \mathrm{~min}$, and quantitatively analyzed using a fluorescence-activated cell sorting (FACS) cytometer (BD Biosciences, Franklin Lakes, USA). Data were analyzed using Flowjo software, version 7.6 (FlowJo LLC, Ashland, OR, USA). At least 3 biological repeats of the cell cycle progression assay were performed for the statistical quantitation of LM3 cell numbers at different cell cycle stages.

Cell migration and invasion assays. The migration and invasion capabilities of liver cancer cells were assessed using the Transwell culture system. For analysis of cell migration, transfected LM3 cells cultured at a density of $1 \times 10^{6} / \mathrm{ml}$ in serum-free medium were placed in the upper chamber, while the lower chamber was filled with culture medium supplemented with $10 \% \mathrm{FBS}$. Following culture at $37^{\circ} \mathrm{C}$ in a humidified incubator supplied with $5 \% \mathrm{CO}_{2}$, cells that had migrated to the lower chamber were incubated with $4 \%$ paraformaldehyde for $10 \mathrm{~min}$, stained with $1 \%$ crystal violet for $10 \mathrm{~min}$ at room temperature, and then counted using a cell counter under an inverted phase-contrast microscope (magnification, x200). To detect the invasive capacity of liver cancer cells, Matrigel ${ }^{\circledR}$ Basement Membrane Matrix (BD Biocoat, BD Biosciences) was used to coat the Transwell chamber at $37^{\circ} \mathrm{C}$ for $2 \mathrm{~h}$. The upper chamber was seeded with liver cancer cells, as aforementioned, and culture medium was placed in the lower chamber. Similar subsequent experimental steps to fix, stain and count the invaded cells were performed as aforementioned in the migration assay protocol. At least 3 biological replicates were performed for each of the migration and invasion experiments.

Western blot analysis. LM3 cells were washed 3 times with PBS and lysed using radioimmunoprecipitation $(50 \mathrm{mM}$ Tris-base, $150 \mathrm{mM} \mathrm{NaCl}, 0.1 \% \mathrm{SDS}, 0.5 \%$ sodium seoxycholate, $1 \%$ Triton $\mathrm{X}-100)$ for total protein extraction. Following determination of protein concentration via a Bicinchoninic Acid assay, $\sim 30 \mu \mathrm{g}$ protein from each group was boiled for 5 min, separated by $12 \%$ SDS-PAGE, and transferred onto a PVDF membrane. The membrane was then blocked at room temperature for $1 \mathrm{~h}$ with $5 \%$ lipid-free milk solution, incubated with primary antibodies and then washed 3 times with TBS + $0.05 \%$ Tween 20 (TBST). The membrane was then incubated with horseradish peroxide-conjugated secondary antibodies (1:10,000; cat. no. 2999, Cell Signaling Technology, Inc., Danvers, MA, USA), washed 3 times with TBST buffer and finally developed using enhanced chemiluminescent solution (Amersham; GE Healthcare, Chicago, IL, USA). The following primary antibodies, purchased from Abcam, were used in this study: Anti-epithelial cadherin (E-cadherin) antibody (cat. no. ab15148, diluted 1:1,000); anti-neural cadherin (N-cadherin) antibody (cat. no. ab18203, diluted 1:1,000); anti-Caspase-3 antibody (cat. no. ab13847, diluted 1:1,000); anti-Cyclin D1 antibody (cat. no. ab134175, diluted 1:1,000); anti-MMP2 antibody (cat. no. ab37150, diluted 1:1,000); anti-GAPDH antibody (cat. no. ab9484, diluted 1:1,000). The relative expression of proteins were quantified using ImageJ (National Institutes of Health, Bethesda, MD, USA) together with 64-bit Java 1.8.0_112.

Statistical analysis. Quantitative data in the present study were statistically analyzed using the SPSS 18.0 software package (SPSS, Inc., Chicago, IL, USA. A Student's t-test was performed to examine the difference between two groups. A one-way analysis of variance followed by Bonferroni method was used to compare the difference between $>2$ groups. $\mathrm{P}<0.05$ was considered to indicate a statistically significant difference.

\section{Results}

Downregulation of IncRNA expression in liver cancer. To examine the potential roles of IncRNAs in liver cancer development and progression, the expression levels of 3 IncRNAs ACTA2-AS1:4, TRPM2-AS1, encoded by the TRPM2 gene, and MUC20-9 encoded by the mucin 20 
A

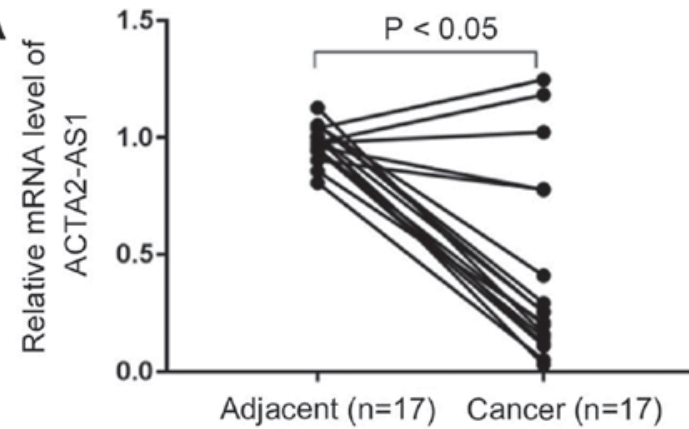

C

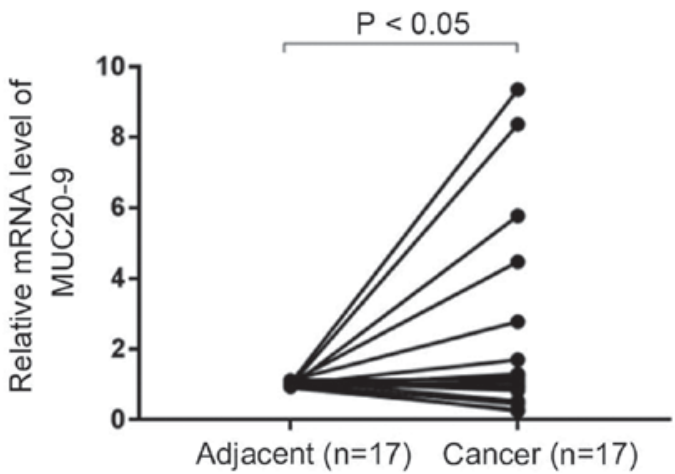

B

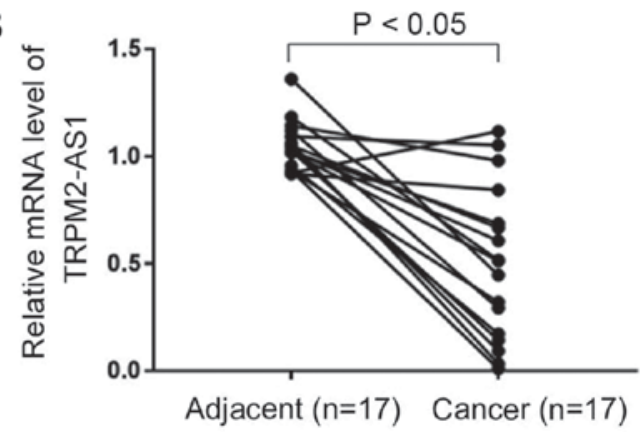

D

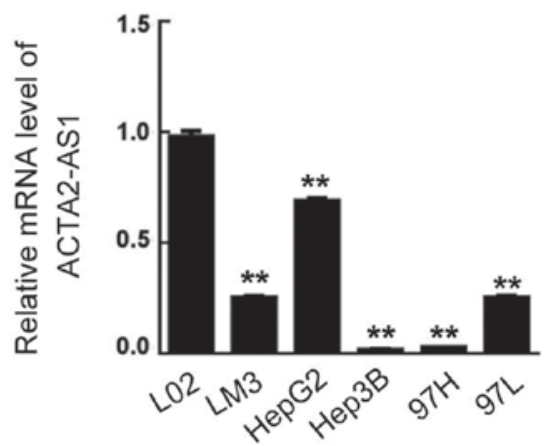

Figure 1. Long non-coding RNA expression in hepatocellular carcinoma biopsies and liver cancer cell lines. The relative expression of (A) ACTA2-AS1:4, (B) TRPM2-AS1 and (C) MUC20-9 in liver cancer biopsies and adjacent normal hepatic tissues was determined by RT-qPCR. (D) The relative expression of ACTA2-AS1:4 in the liver cancer LM3, HepG2, Hep3B, 97H and 97L cell lines was analyzed by RT-qPCR. The normal liver cell line L02 was used as a control. ${ }^{* *} \mathrm{P}<0.01$ vs. LO2, ACTA2, smooth muscle $\alpha$-actin 2; ACTA2-AS1:4, a form of ACTA2-AS1 transcript; TRPM2, transient receptor potential cation channel subfamily M member 2 reverse; MUC20-9, mucin 20, cell surface associated; RT-qPCR, reverse transcription quantitative polymerase chain reaction.

gene, were analyzed by qPCR. As demonstrated in Fig. 1, the expression level of ACTA2-AS1:4 in cancerous tissues collected from 17 patients with liver cancer was significantly downregulated compared with the corresponding adjacent non-cancerous tissues $(\mathrm{P}<0.05$; Fig. 1A). Similarly, the expression of TRPM2-AS1 was suppressed in liver cancer tissues (P<0.05; Fig. 1B). The expression of MUC20-9, however, was increased in liver cancer tissues $(\mathrm{P}<0.05$; Fig. $1 \mathrm{C})$. The differentially altered expression of these 3 lncRNAs in liver cancer tissues suggested that lncRNAs serve distinct roles in liver cancer initiation and progression. For additional verification, the expression of ACTA2-AS1:4 in the normal human liver cell line L02 and 5 liver cancer LM3, HepG2, Hep3B, 97H and 97L cell lines were subsequently analyzed by qPCR; there was a decreased expression of ACTA2-AS1:4 in all five cancerous cell lines compared with the L02 normal liver control cells (Fig. 1D). The downregulation of ACTA2-AS1:4 in both clinical liver cancer biopsies and cancer cell lines suggest that ACTA2-AS1:4 is involved in liver cancer progression.

Suppression of ACTA2-AS1:4 promotes liver cancer cell proliferation. To investigate the potential implication of the decreased expression of ACTA2-AS1:4 in liver cancer cells compared with in normal liver cells, the expression of ACTA2-AS1:4 was knocked down in LM3 cells using siRNA. RT-qPCR analysis confirmed the suppression of ACTA2-AS1:4 expression in LM3 cells following transfection $(\mathrm{P}<0.01$; Fig. 2A). These ACTA2-AS1:4-depleted cells exhibited an increase in cell viability, suggesting that a low level of ACTA2-AS1:4 promotes liver cell proliferation in vivo $(\mathrm{P}<0.01$; Fig. 2B). Furthermore, the percentage of transfected LM3 cells at different stages of the cell cycle was observed. It was indicated that the percentage of ACTA2-AS1:4-depleted cells in S stage was $27.38 \%$, which was markedly increased compared with that in the siRNA NC group (Fig. 2C). The significant increase in S-stage LM3 cells additionally validates the role of ACTA2-AS1:4 in regulating liver cancer proliferation. This suggests that the low expression of ACTA2-AS1:4 in liver cancer cells causes an increase in cells in $\mathrm{S}$ phase, contributing to liver cancer cell proliferation.

Liver cancer cell migration and invasion improved by ACTA2-AS1:4 depletion. The migratory and invasive capabilities of LM3 cells transfected with ACTA2-AS1:4 siRNAs were assessed to provide additional insight into the cellular properties of liver cancer cells. The migration of transfected LM3 cells was markedly increased compared with the NC cells (Fig. 3A and 3B). Similarly, the invasion of transfected cells was increased compared with the NC (Fig. 3C and 3D). These results suggested that in addition to the cell proliferation and cell cycle progression, low expression levels of ACTA2-AS1:4 may also promote liver cancer cell migration and invasion.

Molecular alterations in transfected liver cancer cells. To explore the molecular mechanisms mediating the alteration of multiple cellular properties induced by the suppression of ACTA2-AS1:4, a number of well-known markers associated 

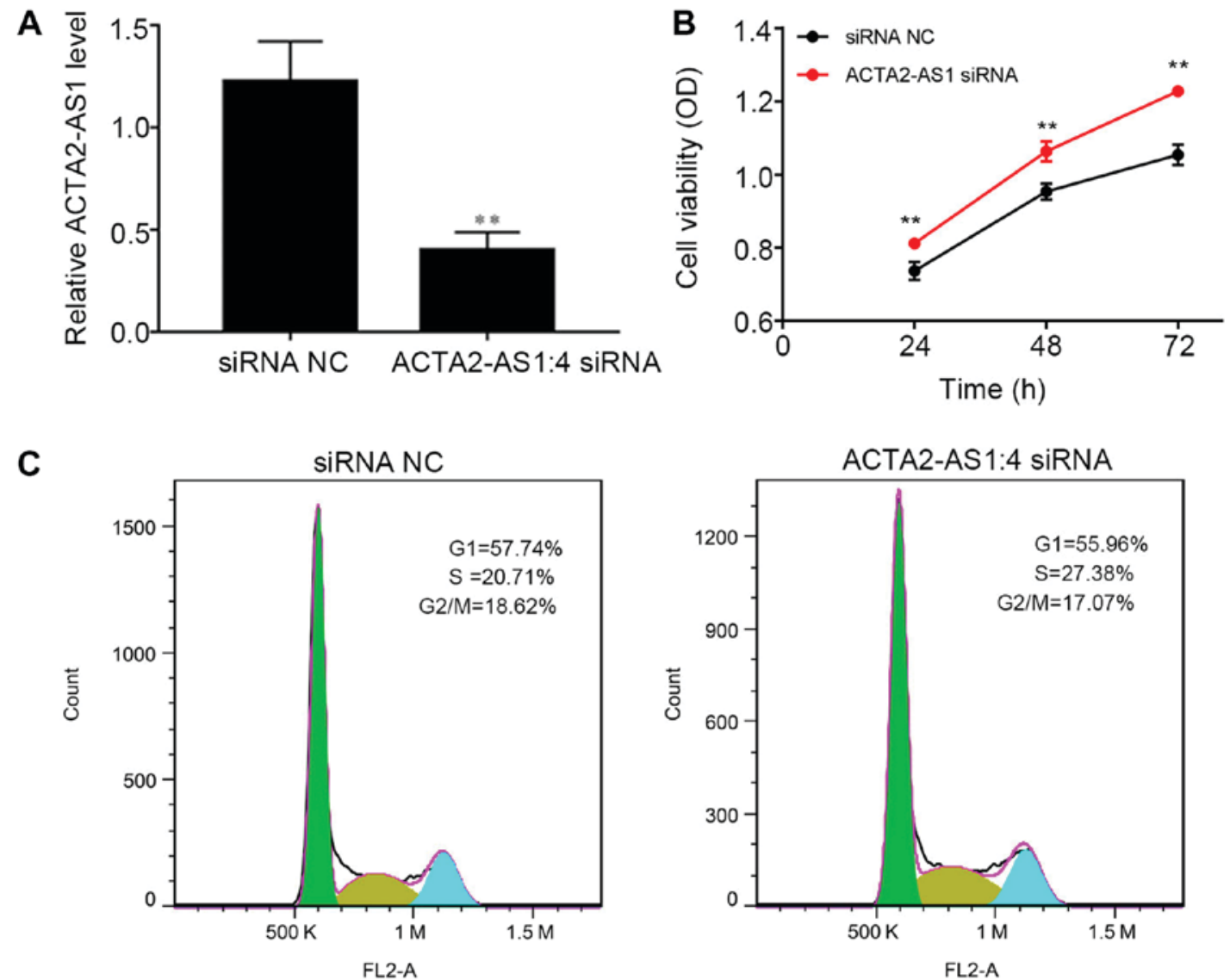

Figure 2. Long non-coding RNA suppression promotes liver cancer cell proliferation. The (A) relative gene expression of ACTA2-AS1:4, (B) cell viability and (C) cell cycle progression of LM3 liver cancer cells transfected with ACTA2-AS1:4 siRNA was determined by reverse transcription quantitative polymerase chain reaction, MTS assay and fluorescence-activated cell sorting analysis, respectively. Non-transfected LM3 cells were used as an NC. ${ }^{* *} \mathrm{P}<0.01$ vs. NC. ACTA2-AS1:4, a form of ACTA2-AS1 transcript; siRNA, small interfering RNA; NC, negative control; OD, optical density.

A

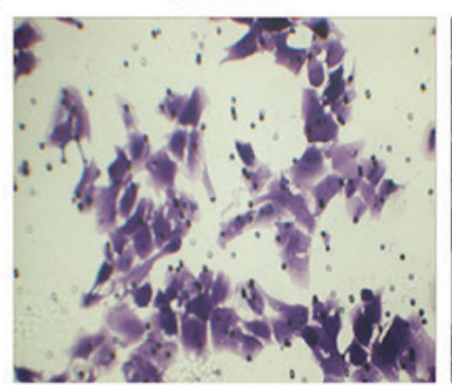

C

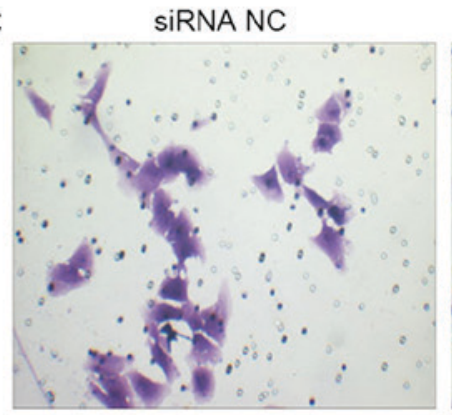

ACTA2-AS1:4 siRNA

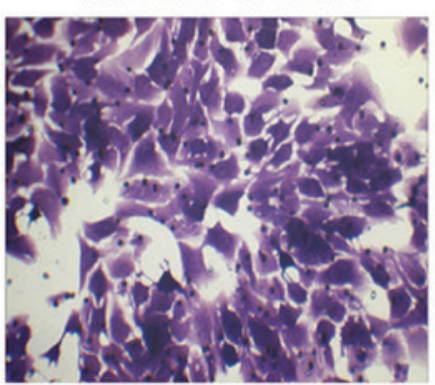

ACTA2-AS1:4 siRNA

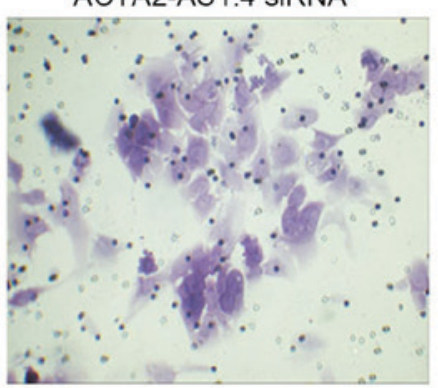

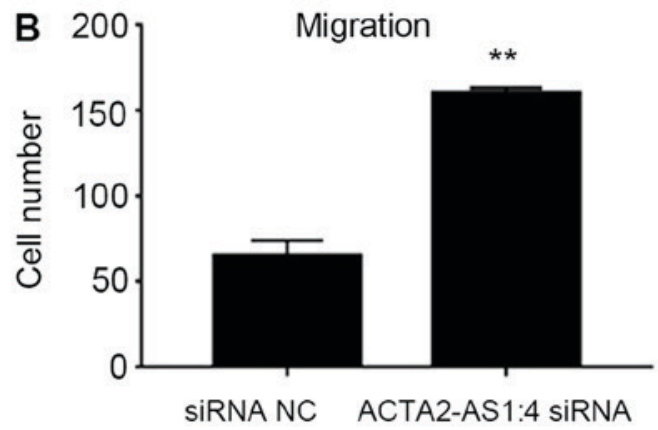

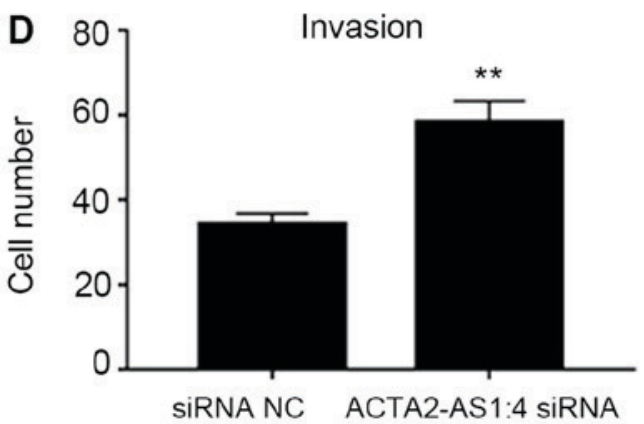

Figure 3. Long non-coding RNA depletion promotes liver cancer cell migration and invasion. LM3 liver cancer cells were transfected with ACTA2-AS1:4 siRNA and the (A) migration of transfected and non-transfected NC cells are presented. (B) Statistical analysis confirmed the difference in migration of transfected cells compared with non-transfected NC cells. (C) Invasion assay of transfected and non-transfected NC cells. (D) Statistical analysis confirmed the difference in migration of transfected cells compared with non-transfected NC cells. " $\mathrm{P}<0.01$ vs. NC. ACTA2-AS1:4, a form of ACTA2-AS1 transcript; siRNA, small interfering RNA; NC, negative control. 
A
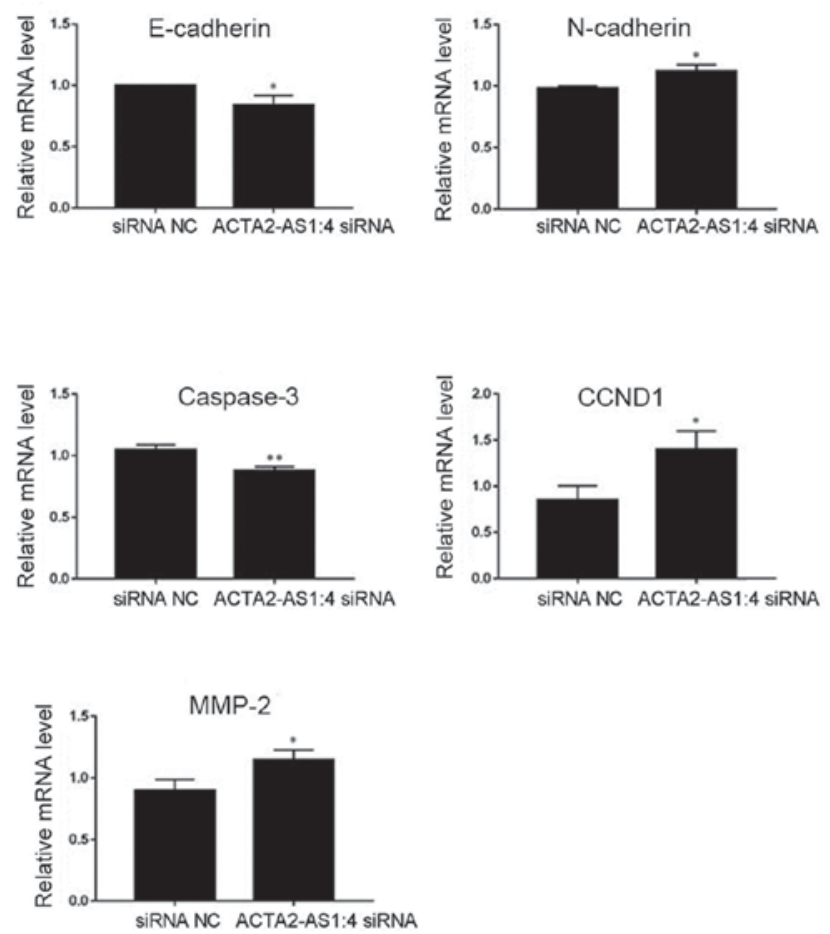

B
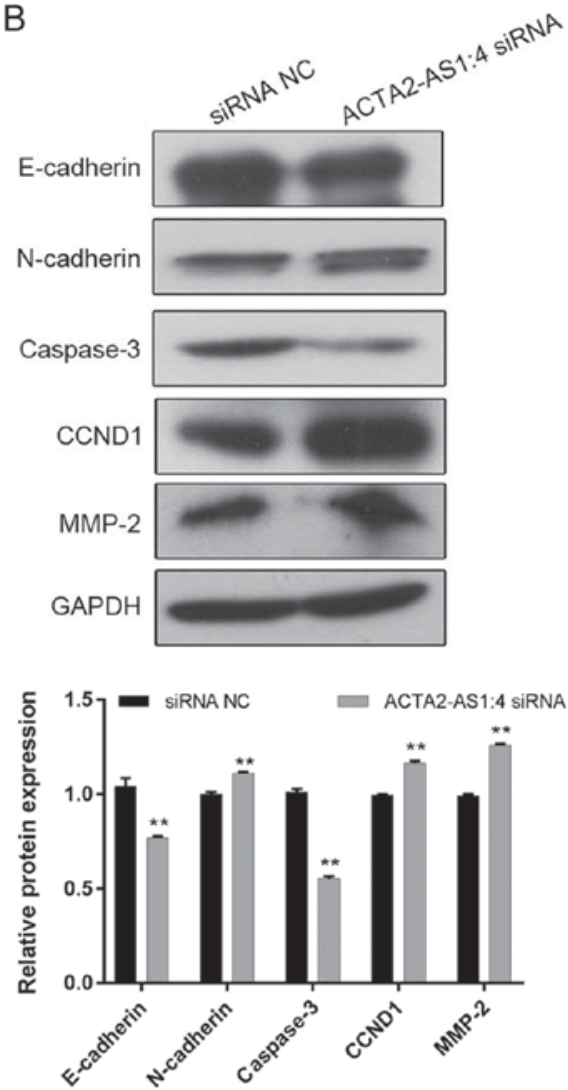

Figure 4. Changes in the mRNA and protein expression of ACTA2-AS1-transfected cells. LM3 cells were transfected with ACTA2-AS1:4 siRNA. (A) The relative mRNA expression of key genes associated with multiple signaling pathways was measured using reverse transcription quantitative polymerase chain reaction. (B) The protein expression of key cellular signaling components was measured using western blot analysis. GAPDH was applied as the internal standard. The proteins investigated were ACTA2-AS1:4, CCND1 and MMP2. NC was used to compare expression levels. "P<0.05 and ${ }^{* *} \mathrm{P}<0.01$ vs. NC. ACTA2-AS1:4, a form of ACTA2-AS1 transcript; E-cadherin, epithelial cadherin; N-cadherin; siRNA, small interfering RNA; CCND1, Cyclin D1; MMP2, matrix metalloproteinase 2; NC, negative control.

with cell proliferation, apoptosis and migration were additionally analyzed in the present study. RT-qPCR assays indicated that the expression of E-cadherin was significantly decreased alongside the depletion of ACTA2-AS1:4 in LM3 cells, while $\mathrm{N}$-cadherin expression increased (Fig. 4A). Additionally, expression of caspase 3, a crucial factor in cell apoptosis, significantly decreased (Fig. 4A) and cyclin D1 (CCND1) and matrix metalloproteinase 2 (MMP2) expression markedly increased following transfection of LM3 cells. The alteration of these gene expression levels was confirmed by the protein abundance of N-cadherin, CCND1 and MMP2 in transfected cells compared with non-transfected control cells (Fig. 4B). Taken together, the statistically significant changes in the expression levels of these key markers suggested that changes in ACTA2-AS1:4 expression serve as regulators of liver cancer progression through multiple downstream molecular pathways.

\section{Discussion}

Noncoding sequences account for $>90 \%$ of the whole genome, according to a bioinformatics analysis following completion of the human genome project (23) At present, a number of functional noncoding sequences have been proved to be involved in various physiological and pathological processes, which include microRNAs and lncRNAs. Increasing evidence demonstrates that lncRNAs are key factors during tumor initiation and progression (12). According to previous data, the effects of lncRNAs have been implicated in tumorigenesis and malignant transformation processes; these include cell proliferation, differentiation, apoptosis, migration and invasion, which are closely associated with cancer metastasis (24). A recent study described the characterization of a novel lncRNA that promotes breast cancer brain metastases (BCBMs) through activation of the c-Jun $\mathrm{N}$-terminal kinase/signal transducer and activator of transcript 3 pathway, providing a potential target in BCBMs that are resistant to conventional therapies (25) The identification of MALAT1, HEIH, HULC and HOTAIR as important regulators of HCC pathology has identified a novel avenue of study for understanding liver cancer development $(26,27)$. However, an estimation based on the ENCODE project has suggested that as many as $>28,000$ lncRNAs may be encoded by the human genome (28), suggesting that the results from the present study pertaining to the roles of lncRNAs in liver cancer pathology remain limited.

To investigate the role of IncRNAs in liver cancer, the expression levels of 3 lncRNAs, ACTA2-AS1:4, TRPM2-AS1 and MUC20-9, known to be implicated in the development of other human cancer types (21), but not hepatic carcinoma, were initially examined. It was observed that ACTA2-AS1:4 and TRPM2-AS1 were upregulated and MUC20-9 was downregulated in liver cancer tissues and cultured liver cancer 
cell lines compared with normal liver tissues and normal cultured cells. This indicated that lncRNAs may serve a role in liver cancer pathology and that each lncRNA in liver cancer cells may serve their own role in cancer progression and/or initiation. Knockdown of noncoding RNA using specific siRNAs has not been widely used to study the in vivo function of IncRNAs (12). To validate the cellular effects of ACTA2-AS1:4 during tumor development and malignant transformation, ACTA2-AS1:4 expression was additionally inhibited via siRNA-mediated knockdown in liver cancer cells in the present study. As expected, the downregulation of ACTA2-AS1:4 markedly promoted liver cancer cell proliferation, altered cell cycle progression and resulted in an increase in cellular migration and invasion, compared with non-transfected controls, suggesting that lncRNAs are critical in various cellular processes associated with liver cancer initiation, development and metastasis. Finally, the pleiotropic cellular functions of ACTA2-AS1:4 in liver cancer cells were additionally verified by the changes in the expression of multiple key regulatory proteins involved in different signaling pathways. In the present study, significant changes in ACTA2-AS1:4 expression and cellular alterations induced by ACTA2-AS1:4 knockdown provided direct evidence of the role of ACTA2-AS1:4 in promoting complex cellular processes involved in liver cancer development. These data are similar to other cancer-associated lncRNAs described previously (12). The RT-qPCR assays performed in the present study indicated that the expression of E-cadherin was significantly downregulated alongside the decrease of ACTA2-AS1:4 expression in liver cancer LM3 cells, while the $\mathrm{N}$-cadherin expression exhibited marked increased; therefore, we hypothesized that ACTA2-AS1:4 expression may affect the epithelial-mesenchymal transition of the liver cancer cells. These results also suggested that ACTA2-AS1:4 may be explored as a novel biomarker for early liver cancer detection and development of novel targeted therapies for patients with liver cancer.

Notably, the present study is limited due to unclear molecular mechanisms underlying the pro-cancer function of ACTA2-AS1:4 in liver cancer cells, which warrants additional investigation. The interaction of lncRNAs with functional proteins serves as one of the major molecular mechanisms by which lncRNAs perform their physiological and pathological roles (12). For example, the lncRNA breast cancer anti-estrogen resistance 4 was identified to regulate breast cancer metastasis through chemokine-induced interaction with two transcription factors, resulting in p300-dependent histone acetylation and activation of the Hedgehog/zinc finger protein GLI2 transcriptional program, which finally promotes cancer cell migration and invasion (29). Therefore, it is reasonable to hypothesize that future screening of proteins interacting with ACTA2-AS1:4 during liver cancer progression may provide more information about underlying molecular processes. Potentially, and more importantly, future studies of epigenetic markers may also provide novel perspectives on the molecular mechanisms involved in the role of ACTA2-AS1:4 during liver cancer progression, considering the association of lncRNAs with epigenetic regulation described previously (12).

Furthermore, how ACTA2-AS1:4 expression was downregulated in liver cancer cells is an additional key topic for future study, as comprehensive understanding of the regulation of cancer-associated lncRNAs expression may also provide novel targets for early cancer diagnosis and treatment. A previous study revealed that the lncRNA maternally expressed gene 3 may be regulated by $\mathrm{CpG}$ hypomethylation at its promoter region and also histone-H3 lysine-4 trimethylation in response to menin, (30), suggesting that epigenetic regulation may be a key method of modulating lncRNAs expression during cancer development and progression. Combined with the regulation of an epigenetic-associated pathway by lncRNAs, as aforementioned, it is possible that alterations in ACTA2-AS1:4 expression may affect the induction of pathways, such as the epigenetic-associated pathway, which in turn may regulate its own transduction. We propose that such a feedback loop may be an essential component of the complicated networks involved in liver cancer pathology.

Taken together, the present study describes the identification of a novel liver cancer-associated lncRNA ACTA2-AS1:4:4, which is markedly downregulated in liver cancer tissues and cell lines. The siRNA-mediated inhibition of ACTA2-AS1:4:4 promoted liver cancer cell proliferation, cell cycle progression, migration and invasion, supported by changes in the expression of key components of tumor-associated signaling pathways. These data provide a basis for the application of lncRNAs in the early detection of liver cancer, and potentially the development of novel treatment strategies.

\section{Acknowledgements}

Not applicable.

\section{Funding}

Not funding was received.

\section{Availability of data and materials}

All data generated or analyzed during this study are included in this published article.

\section{Authors' contribution}

R-JZ performed the measurements, analyzed and interpreted data. H-ZL made substantial contributions to conception and design, and were involved in drafting, revising the manuscript and interpreting all data. All authors read and approved the final manuscript.

\section{Ethics approval and consent to participate}

The study proceeded following the receipt of written informed consent from the patients, and tissue collection and subsequent experiments were approved by the Ethics Committee of the Sun Yat-sen University and the Fifth Affiliated Hospital of Guangzhou Medical University.

\section{Patient approval for publication}

Not applicable. 


\section{Competing interests}

The authors declare that they have no competing interests.

\section{References}

1. Sia D, Villanueva A, Friedman SL and Llovet JM: Liver cancer cell of origin, molecular class, and effects on patient prognosis. Gastroenterology 152: 745-761, 2017.

2. Castelli G, Pelosi E and Testa U: Liver cancer: Molecular characterization, clonal evolution and cancer stem cells. Cancers (Basel) 9: E127, 2017.

3. Ghouri YA, Mian I and Rowe JH: Review of hepatocellular carcinoma: Epidemiology, etiology, and carcinogenesis. J Carcinog 16: 1, 2017.

4. Roth GS and Decaens T: Liver immunotolerance and hepatocellular carcinoma: Patho-physiological mechanisms and therapeutic perspectives. Eur J Cancer 87: 101-112, 2017.

5. Lin C and Yang L: Long noncoding RNA in cancer: Wiring signaling circuitry. Trends Cell Biol 28: 287-301, 2018.

6. Prensner JR and Chinnaiyan AM: The emergence of lncRNAs in cancer biology. Cancer Discov 1: 391-407, 2011.

7. Cloutier SC, Wang S, Ma WK, Al Husini N, Dhoondia Z, Ansari A, Pascuzzi PE and Tran EJ: Regulated formation of IncRNA-DNA hybrids enables faster transcriptional induction and environmental adaptation. Mol Cell 62: 148, 2016.

8. Yoon JH, Abdelmohsen K and Gorospe M: Post-transcriptional gene regulation by long noncoding RNA. J Mol Biol 425 3723-3730, 2013.

9. Szcześniak MW and Makałowska I: lncRNA-RNA interactions across the human transcriptome. PLoS One 11: e150353, 2016.

10. Li JH, Liu S, Zheng LL, Wu J, Sun WJ, Wang ZL, Zhou H, Qu LH and Yang JH: Discovery of protein-lncRNA interactions by integrating large-scale CLIP-seq and RNA-Seq datasets. Front Bioeng Biotechnol 2: 88, 2015.

11. Zampetaki A, Albrecht A and Steinhofel K: Long Non-coding RNA structure and function: Is there a link? Front Physiol 9: 1201, 2018.

12. Mehra M and Chauhan R: Long noncoding RNAs as a key player in hepatocellular carcinoma. Biomarkers Cancer 9: 1179299X17737301, 2017

13. Huarte M, Guttman M, Feldser D, Garber M, Koziol MJ, Kenzelmann-Broz D, Khalil AM, Zuk O, Amit I, Rabani M, et al: A large intergenic noncoding RNA induced by p53 mediates global gene repression in the p53 response. Cell 142: 409-419, 2010.

14. Du Y, Kong G, You X, Zhang S, Zhang T, Gao Y, Ye L and Zhang $X$ : Elevation of highly up-regulated in liver cancer (HULC) by hepatitis B Virus X protein promotes hepatoma cell proliferation via down-regulating $\mathrm{p} 18$. J Biol Chem 287: 26302-26311, 2012.

15. Li H, An J, Wu M, Zheng Q, Gui X, Li T, Pu H and Lu D: LncRNA HOTAIR promotes human liver cancer stem cell malignant growth through downregulation of SETD2. Oncotarget 6: 27847-27864, 2015 .
16. Conigliaro A, Costa V, Lo Dico A, Saieva L, Buccheri S, Dieli F, Manno M, Raccosta S, Mancone C, Tripodi M, et al: CD90+ liver cancer cells modulate endothelial cell phenotype through the release of exosomes containing H19 lncRNA. Mol Cancer 14: $155,2015$.

17. Yang F, Zhang L, Huo XS, Yuan JH, Xu D, Yuan SX, Zhu N, Zhou WP, Yang GS, Wang YZ, et al: Long noncoding RNA high expression in hepatocellular carcinoma facilitates tumor growth through enhancer of zeste homolog 2 in humans. Hepatology 54: 1679-1689, 2011.

18. Wu M, Lin Z, Li X, Xin X, An J, Zheng Q, Yang Y and Lu D: HULC cooperates with MALAT1 to aggravate liver cancer stem cells growth through telomere repeat-binding factor 2 . Sci Rep 6: 36045, 2016.

19. Cooper K and Brown S: ACTA2 mutation and postpartum hemorrhage: A case report. BMC Med Genet 18: 143, 2017.

20. Désert R, Mebarki S, Desille M, Sicard M, Lavergne E, Renaud S, Bergeat D, Sulpice L, Perret C, Turlin B, et al: 'Fibrous nests' in human hepatocellular carcinoma express a Wnt-induced gene signature associated with poor clinical outcome. Int J Biochem Cell Biol 81: 195-207, 2016.

21. Zhang L, Zhou XF, Pan GF and Zhao JP: Enhanced expression of long non-coding RNA ZXF1 promoted the invasion and metastasis in lung adenocarcinoma. Biomed Pharmacother 68: 401-407, 2014.

22. Livak KJ and Schmittgen TD: Analysis of relative gene expression data using real-time quantitative PCR and the 2 (-Delta Delta C(T)) method. Methods 25: 402-408, 2001.

23. Edwards YJ, Walter K, Mc-Ewen G, Vavouri T, Kelly KA, Abnizova I, Woolfe A, Goode DK, Goodson M, North P, et al: Characterisation of conserved non-coding sequences in vertebrate genomes using bioinformatics, statistics and functional studies. Comp Biochem Physiol Part D Genomics Proteomics 1: 46-58, 2006.

24. Bhan A, Soleimani M and Mandal SS: Long noncoding RNA and cancer: A new paradigm. Cancer Res 77: 3965-3981, 2017.

25. Wang S, Liang K, Hu Q, Li P, Song J, Yang Y, Yao J, Mangala LS, Li C, Yang W, et al: JAK2-binding long noncoding RNA promotes breast cancer brain metastasis. J Clin Invest 127: 4498-4515, 2017.

26. Geng YJ, Xie SL, Li Q, Ma J and Wang GY: Large Intervening Non-Coding RNA HOTAIR is associated with hepatocellular carcinoma progression. J Int Med Res 39: 2119-2128, 2011.

27. He Y, Meng XM, Huang C, Wu BM, Zhang L, Lv XW and Li J: Long noncoding RNAs: Novel insights into hepatocelluar carcinoma. Cancer Lett 344: 20-27, 2014.

28. Tragante V, Moore JH and Asselbergs FW: The ENCODE project and perspectives on pathways. Genet Epidemiol 38: 275-280, 2014.

29. Xing Z, Lin A, Li C, Liang K, Wang S, Liu Y, Park PK, Qin L, Wei Y, Hawke DH, et al: lncRNA directs cooperative epigenetic regulation downstream of chemokine signals. Cell 159: 1110-1125, 2014

30. Modali SD, Parekh VI, Kebebew E and Agarwal SK: Epigenetic regulation of the lncRNA MEG3 and its target c-MET in pancreatic neuroendocrine tumors. Mol Endocrinol 29: 224-237, 2015. 